\title{
A flexible framework for modeling multiple solid, liquid and gaseous phases
}

\section{in batteries and fuel cells}

\author{
Jonathan P. Neidhardt ${ }^{1,2}$, Thomas Jahnke ${ }^{1}$, David N. Fronczek ${ }^{1}$, Timo Danner ${ }^{1,2}$, Birger Horstmann ${ }^{1,2}$, \\ and Wolfgang G. Bessler ${ }^{1,2}$ \\ ${ }^{1)}$ German Aerospace Center (DLR), Institute of Technical Thermodynamics \\ Pfaffenwaldring 38-40, 70569 Stuttgart \\ ${ }^{2)}$ Institute of Thermodynamics and Thermal Engineering (ITW), Stuttgart University, Pfaffenwaldring \\ 6, 70550 Stuttgart \\ jonathan.neidhardt@dlr.de
}

Multi-phase management is crucial for performance and durability of electrochemical cells such as batteries and fuel cells. Here we present a generic framework [1] for describing the two-dimensional spatiotemporal evolution of gaseous, liquid and solid phases, as well as their interdependence with interfacial (electro-)chemistry and structure in a continuum description. The modeling domain consists of up to seven layers (current collectors, channels, electrodes, separator/membrane), each of which can consist of an arbitrary number of bulk phases (gas, liquid, solid) and connecting interfaces (two-phase or multi-phase boundaries). Bulk and interfacial chemistry is described using global or elementary kinetic reactions. Multi-phase management is coupled to mass and charge transport within bulk phases. The functionality and flexibility of this framework is demonstrated using four application areas in the context of post-lithium-ion batteries and fuel cells:

(1) Lithium-sulfur (Li-S) batteries are characterized by a complex sulfur redox chemistry. Based on eight chemical reactions between four solid phases and eight electrolyte-dissolved species, we were able to predict the discharge behavior.

(2) The functionality of lithium-oxygen (Li-O) cells is based on the presence of four phases in three different states of aggregation at the positive electrode. The performance of the battery is limited by oxygen transport in the electrolyte. If $\mathrm{Li}_{2} \mathrm{O}_{2}$ is concentrated near the cathode-gas interface it can block the pores of the cathode and the discharge stops prematurely.

(3) Nickel-based solid oxide fuel cell (SOFC) anodes are sensitive towards formation of nickel oxide. The formation of $\mathrm{NiO}$ was shown to occur predominantly in the composite anode close to the solid electrolyte and close to the channel outlet.

(4) Water management of polymer electrolyte membrane fuel cells (PEFC) is of high importance for good performance. Spatiotemporal simulation of water saturation in the PEFC cathode shows flooding, which eventually inhibits the cell function.

The present methodology provides a useful tool for understanding and optimizing multi-phase management in electrochemical cells.

References:

1. J.P. Neidhardt, D.N. Fronczek, T. Jahnke, T. Danner, B. Horstmann and W.G. Bessler, J. Electrochem. Soc., submitted (2012) 